\title{
Two-Site Model for Human Dental Enamel Dissolution in EDTA
}

\author{
J. L. FOX, W. I. HIGUCHI, M. FAWZI, R. C. HWU, and J. J. HEFFERREN \\ College of Pharmacy, University of Michigan, Ann Arbor, Michigan 48104, USA and \\ the American Dental Association, Chicago, Illinois
}

Dissolution of tooth enamel and of hydroxyapatite (HAP) in buffered solutions of ethylenediaminetetraacetic acid (EDTA) has been studied in this laboratory by the physical model approach. To quantitatively explain the experimental results, it was necessary to develop a model incorporating two different types of crystalline sites for dissolution.

In a previous study using a one-site diffusion layer (D-L) model similar to that described by Higuchi et al ( $J$ Dent Res, 44: 330, 1965), theoretical predictions were generated for the dissolution of HAP in buffered EDTA solutions over a range of $\mathrm{pH}$ and EDTA concentration. The D-L model predicted linearly increasing rates of dissolution with increases in EDTA. Experiments with powdered TVA hydroxyapatite (Tennessee Valley Authority, Report No. 678, Wilson Dam Alabama, November 6, 1956), however, showed that EDTA decreased dissolution rates at a $\mathrm{pH}$ of 3 or 4 while at a $\mathrm{pH}$ of 5 or 6 it did not change the rates appreciably from the controls. In all instances, rates in the presence of EDTA were much less than predicted. It was concluded that the sites responsible for dissolution of TVA hydroxyapatite were greatly inhibited by EDTA. Powdered samples of NBS $^{\text {a }}$ hydroxyapatite (AvNIMELECH, Isr $J$ Chem, 6: 375, 1968) and of enamel used in similar studies indicated that there was some inhibition by EDTA, but to a much lesser degree than in the TVA studies. These studies, along with scanning electron microscope studies on block enamel (FAwzI ET AL, in press) which strongly imply the presence of two sites, led to the following model for block enamel. The enamel is assumed to consist of a matrix of HAP crystals with adequate intercrystalline spaces for the permeation of aqueous solutions. Each of these crystals, then is assumed to have two different types of sites on its surface. One of the sites is assumed to be inhibited by EDTA like the predominant site of TVA hydroxyapatite, while the other site is assumed to be unaffected by EDTA. This second site, however, is not necessarily in instantaneous equilibrium with its surroundings. The rate of release of material from this site in the initial model presented here is assumed to be given by the expression: $R_{a}=$

This research was supported by USPHS Grant DE01830 and Training Grant DE-00204 from the $\mathrm{Na}$ tional Institute of Dental Research, National Institutes of Health, Bethesda, Md.

Received for publication October 19, 1973.

- National Bureau of Standards.

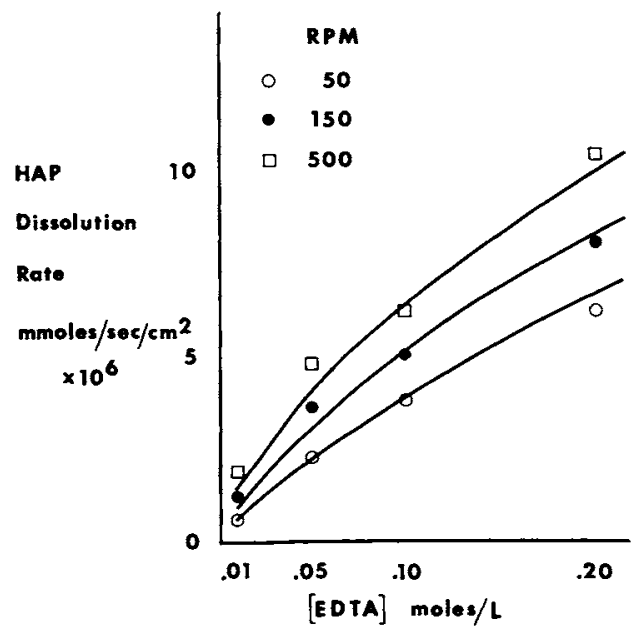

Comparison of experimental dissolution rates with theoretical predictions of two-site model.

$k_{\mathrm{s}}{ }^{*}\left(C_{\mathrm{s}}-C(x)\right)$, where $C_{\mathrm{s}}$ is the equilibrium solubility characteristic of the site and $C(x)$ is the concentration at a given point in the system. To simplify the mathematics, a single reaction was used to approximate the chemical equilibriums of the system at a $\mathrm{pH}$ of 6 :

$$
\text { HAP + EDTA = COMPLEX. }
$$

Since the studies on this model are being car. ried out with a rotating disk system, the effective D-L thickness can be calculated as a function of diffusivity, viscosity, and rotation speed. The Fick's second law equation for the various species, the equilibrium relationship, and the expression for $R_{s}$ were combined and solved numerically for various combinations of EDTA and rotation speed. The theoretical curves and experimental points are shown in the illustra. tion.

A noteworthy consequence of this model is that a zone of dissolution (rather than surface dissolution) is predicted, which is also in good agreement with experimental observations. A more detailed development of the model, now in progress, should significantly advance the understanding of enamel demineralization and caries formation. 